\title{
Visualization of Sphere and Horosphere Packings Related to Coxeter Tilings by Simply Truncated Orthoschemes with Parallel Faces
}

\author{
Visualization of Sphere and Horosphere Packings \\ Related to Coxeter Tilings by Simply Truncated \\ Orthoschemes with Parallel Faces
}

\section{ABSTRACT}

In this paper we describe and visualize the densest ball and horoball packing configurations to the simply truncated 3dimensional hyperbolic Coxeter orthoschemes with parallel faces, using the results of [24]. These beautiful packing arrangements describe and show the very interesting structure of the mentioned orthoschemes and the corresponding Coxeter reflection group. We use the Beltrami-CayleyKlein ball model of 3-dimensional hyperbolic space $\mathbb{H}^{3}$, the images were made by the Python programming language.

Key words: Coxeter group, horosphere, hyperbolic geometry, packing, tilings

MSC2010: 52C17 52C22 52B15

\section{Introduction}

Visualization of mathematical problems is not only a representation of specific objects or an approach in the teaching process, but also plays an important role in understanding the problem and developing solution steps. It can be shown the deeper context of the problem and the possibilities to move forward.

In hyperbolic spaces $\mathbb{H}^{n}$ for $2 \leq n \leq 9$, the known densest ball and horoball configurations are derived by Coxeter simplex tilings, generated by reflections in the simplex hyperplanes [5]. In the former papers, they do not have parallel faces.

Vizualizacija pakiranja sfera i horosfera povezanih s Coxeterovim popločavanjem krnjim ortoshemama paralelnih strana

\section{SAŽETAK}

U ovom radu opisujemo i vizualiziramo najgušće konfiguracije pakiranja sfera i horosfera na krnjim 3dimenzionalnim hiperboličnim Coxeterovim ortoshemama s paralelnim stranama, koristeći rezultate [24]. Ovi lijepi rasporedi pakiranja opisuju i pokazuju vrlo zanimljivu strukturu spomenutih ortoshema i odgovarajuće Coxeterove zrcalne grupe. Koristimo sferni Beltrami-Cayley-Kleinov model 3-dimenzionalnog hiperboličnog prostora $\mathbb{H}^{3}$. Slike su izrađene programskim jezikom Python.

Ključne riječi: Coxeterova grupa, horosfera, hiperbolična geometrija, pakiranje, popločavanje

In periodic ball or horoball packings, the local density described below can be extended to the entire hyperbolic space and it is related to the simplicial density function that we generalized in [19] and [20]. In this paper, we shall use such definition of packing density by [24].

A Coxeter simplex in $\overline{\mathbb{H}}^{n}$ has dihedral angles either integral submultiples of $\pi$ or zero. Thus, the group generated by reflections in the simplex side hyperplanes is isometry group of $\mathbb{H}^{n}$ with the Coxeter simplex as fundamental domain. Hence the group gives regular tessellations. We note here that the Coxeter groups are finite for $\mathbb{S}^{n}$, and infinite for $\mathbb{E}^{n}$ or $\overline{\mathbb{H}}^{n}$ [1, 5, 7, 8, 9, 17, 23].

There are non-compact Coxeter simplices in $\overline{\mathbb{H}}^{n}$ with ideal vertices in $\partial \mathbb{H}^{n}$, however, only for dimensions $2 \leq n \leq 9$; 
and only a finite number of them exists in dimensions $n \geq 3$, see Johnson et al. [9] and Kellerhals [10]. Such simplices are the most elementary building blocks of hyperbolic manifolds, the volume of which is an important topological invariant.

The simplicial packing density upper bound $d_{3}(\infty)=(1+$ $\left.\frac{1}{2^{2}}-\frac{1}{4^{2}}-\frac{1}{5^{2}}+\frac{1}{7^{2}}+\frac{1}{8^{2}}-\frac{1}{10^{2}}-\frac{1}{11^{2}}+\ldots\right)^{-1}=0.85327 \ldots$ cannot be achieved by packing regular balls, instead it is realized by horoball packings of $\overline{\mathrm{H}}^{3}$, the regular ideal simplex tiles. More precisely, the centres of horoballs in $\partial \overline{\mathbb{H}}^{3}$ lie at the vertices of the ideal regular Coxeter simplex tiling with Schläfli symbol $(3,3,6)$, see [2, 3, 4, 6, 18].

In [11] we have proved that this optimal horoball packing configuration in $\mathbb{H}^{3}$ is not unique. We gave asymptotic Coxeter packings by horoballs of different types, that have different relative densities with respect to the fundamental domain, yielding the Böröczky-Florian-type simplicial upper bound [4].

Furthermore, in [19, 20] we have found that, by allowing horoballs of different types at each vertex of a totally asymptotic simplex and generalizing the simplicial density function to $\overline{\mathbb{H}}^{n}$ for $(n \geq 2)$, the Böröczky-type density upper bound is not valid for the fully asymptotic simplices for $n \geq 4$. For example, in $\overline{\mathbb{H}}^{4}$ the locally optimal simplicial packing density is $0.77038 \ldots$, higher than the Böröczkytype density upper bound of $d_{4}(\infty)=0.73046 \ldots$ using horoballs of a single type. However, these ball packing configurations are only locally optimal and cannot be extended to the entirety of the ambient space $\overline{\mathbb{H}}^{n}$. In [12] we found seven horoball packings of Coxeter simplex tilings in $\overline{\mathbb{H}}^{4}$ that yield densities of 0.71645 , counterexamples to L. Fejes Tóth's conjecture stated in his foundational book Regular Figures [6, p. 323].

In [24], we reported [13] and [14] and considered the Coxeter tilings in $\mathbb{H}^{3}$ where the generating orthoscheme was a simple truncated one with some parallel faces i.e. their dihedral angle is zero (symbol $\infty$ ). Here we studied the Coxeter tilings with Schläfli symbol $(\infty, q, r, \infty)$ (see Fig. 1. second graph). We determined their optimal ball and horoball packings, proved that the densest packing was realized at tilings $(\infty, 3,6, \infty)$, and $(\infty ; 6 ; 3 ; \infty)$ with density $\approx 0.8413392$, see Fig.1, 12, 19 and [20, 21, 22] and [14, 15, 16] for further connections.

\section{Basic Notions}

For the computations and visualization, we use the projective model of the hyperbolic space $\mathbb{H}^{3}[1,16,23]$. The model is defined in general in the pseudo-Euclidean or Lorentz space $\mathbb{E}^{1, n}$ with signature $(1, n)$, i.e. consider real vector space $\mathbf{V}^{n+1}$ equipped with the bilinear form:

$\langle\mathbf{x}, \mathbf{y}\rangle=-x^{0} y^{0}+x^{1} y^{1}+\cdots+x^{n} y^{n}$

and the following equivalence relation:

$\mathbf{x}\left(x^{0}, \ldots, x^{n}\right) \sim \mathbf{y}\left(y^{0}, \ldots, y^{n}\right) \Leftrightarrow \exists c \in \mathbb{R} \backslash\{0\}: \mathbf{y}=c \cdot \mathbf{x}$

to interpret the same point $[x]=[y]$ of $\mathbb{H}^{n}$. The following quadratic form (as a cone in $V^{n+1}$ ):

$Q=\left\{[\mathbf{x}] \in \mathcal{P}^{n} \mid\langle\mathbf{x}, \mathbf{x}\rangle=0\right\}=: \partial \mathbb{H}^{n}$

defines the boundary points (at infinity), the inner or proper points of $\mathbb{H}^{n}$ (for them $\langle\mathbf{x}, \mathbf{x}\rangle<0$ ), and the points lying outside of $Q$ are outer points of $\mathbb{H}^{n}$ (for them $\langle\mathbf{x}, \mathbf{x}\rangle>0$ ). We can also define a linear polarity between the points and hyperplanes: the polar hyperplane $(a)$ of a point $[\mathbf{x}] \in \mathcal{P}^{n}$ is $\operatorname{Pol}(\mathbf{x}):=(a)=\left\{[\mathbf{y}] \in \mathcal{P}^{n} \mid\langle\mathbf{x}, \mathbf{y}\rangle=0\right\}$, and hence $\mathbf{x} \in \mathbf{V}^{n+1}$ is incident with $a \in V_{n+1}$ iff $\mathbf{x} a=0$. In this projective model, we can define a metric structure related to the above bilinear form, where for the distance of two proper (inner) points:

$\cosh \left(\frac{d(\mathbf{x}, \mathbf{y})}{k}\right)=\frac{-\langle\mathbf{x}, \mathbf{y}\rangle}{\sqrt{\langle\mathbf{x}, \mathbf{x}\rangle\langle\mathbf{y}, \mathbf{y}\rangle}}$,

(at present we may choose $k=1$ ).

This corresponds to the distance formula in the well-known Beltrami-Cayley-Klein model of $\mathbb{H}^{n}$ of constant curvature $K=-k^{2}=-1$. We do not detail the analogous angle metric for the dual form space $V_{n+1}$ that present hyperfaces and - cos expresses their angles of normal vectors (through complex numbers), like in the spherical plane and space [1, 9, 16, 17, 24](see also sect 3).

For a general projective coordinate simplex $A_{0} A_{1} A_{2} A_{3}$ we use the vector basis $\mathbf{a}_{0}, \mathbf{a}_{1}, \mathbf{a}_{2}, \mathbf{a}_{3} \in \mathbf{V}^{4}$; for its faces $b^{0}, b^{1}, b^{2}, b^{3}$ stand $b^{0}, b^{1}, b^{2}, b^{3} \in V_{4}$ with $\mathbf{a}_{i} b^{j}=\delta_{i}^{j}$, the Kronecher symbol (Einstein convention). A symmetric linear polarity, i.e. plane $\longrightarrow$ point mapping: $V \ni u \longrightarrow$ $\mathbf{u} \in \mathbf{V}^{4}$ will be defined by $b^{i} \longrightarrow B^{i}, b^{i} \longrightarrow b^{i j} \mathbf{a}_{j}$ with $b^{i j}=b^{j i}(i, j \in\{0,1,2,3\})$, equivalent with a scalar product $\langle u, v\rangle \longrightarrow \mathbb{R},\left\langle b^{i} u_{i}, b^{j} v_{j}\right\rangle=b^{i r} u_{i} \mathbf{a}_{r} b^{j} v_{j}=u_{i} b^{i r} \delta_{r}^{j} v_{j}=$ $u_{i} b^{i j} v_{j}$.

If the polarity is invertible, i.e $\left(b^{i j}\right)^{-1}=a_{i j}=\left\langle\mathbf{a}_{i}, \mathbf{a}_{j}\right\rangle$, as for $\mathbb{H}^{3}$, then forms (normal vectors of planes) and vectors can be "identified", as later on a polar plane $\longleftrightarrow$ with its pole point in $\mathbb{H}^{3}$. 


\section{The structure of truncated asymptotic or- thoscheme}

Our aim is to visualize the truncated simply asymptotic orthoschemes that contain parallel faces in $\mathbb{H}^{3}$. This orthoschemes are represented by their Coxeter graphs (see Fig.1-2), where the angle parameters $p, q, r$ satisfy the inequalities $\frac{\pi}{p}+\frac{\pi}{q}<\frac{\pi}{2}$ and $\frac{\pi}{q}+\frac{\pi}{r} \geq \frac{\pi}{2}$.

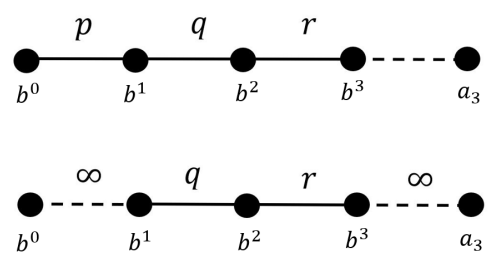

Figure 1: Coxeter graphs of truncated asymptotic orthoscheme

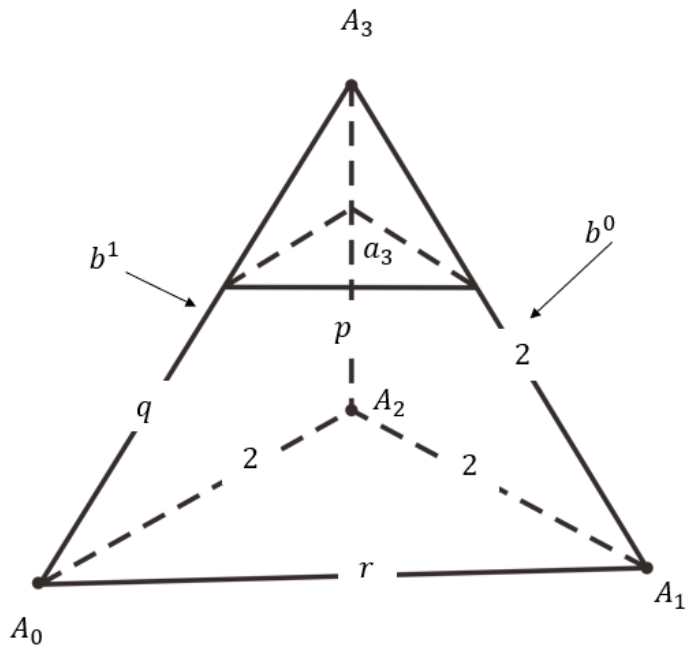

Figure 2: A simply truncated orthoscheme with polar $a_{3}$ of $A_{3} \frac{\pi}{p}+\frac{\pi}{q}<\frac{\pi}{2}, \frac{\pi}{q}+\frac{\pi}{r} \geq \frac{\pi}{2}$

First, we will study the truncated orthoschemes that have the corresponding singular Coxeter-Schläfli matrix as follows (e.g from [7, 17]):

$C=\left[\begin{array}{ccccc}1 & -\cos \left(\frac{\pi}{p}\right) & 0 & 0 & 0 \\ -\cos \left(\frac{\pi}{p}\right) & 1 & -\cos \left(\frac{\pi}{q}\right) & 0 & 0 \\ 0 & -\cos \left(\frac{\pi}{q}\right) & 1 & -\cos \left(\frac{\pi}{r}\right) & 0 \\ 0 & 0 & -\cos \left(\frac{\pi}{r}\right) & 1 & c_{4} \\ 0 & 0 & 0 & c_{4} & 1\end{array}\right]$ where the constant $c_{4}$ can be uniquely determined by the zero determinant condition .

$c_{4}=-\sqrt{\frac{1+\cos ^{2}\left(\frac{\pi}{p}\right) \cos ^{2}\left(\frac{\pi}{r}\right)-\cos ^{2}\left(\frac{\pi}{p}\right)-\cos ^{2}\left(\frac{\pi}{q}\right)-\cos ^{2}\left(\frac{\pi}{r}\right)}{1-\cos ^{2}\left(\frac{\pi}{p}\right)-\cos ^{2}\left(\frac{\pi}{q}\right)}}$.

In our case, there are two parallel faces that meet in an ideal point. That means the dihedral angle between these two hyperplanes is equal to 0 . Therefore, we assume that these two hyperplanes are $b^{0}$ and $b^{1}$. Thus, their dihedral angle is $w^{01}=\frac{\pi}{p} \rightarrow 0$, if $p$ tends to $\infty$, then Coxeter-Schläfli matrix (2) would change to the following form

$C^{\prime}=\left[\begin{array}{ccccc}1 & -1 & 0 & 0 & 0 \\ -1 & 1 & -\cos \left(\frac{\pi}{q}\right) & 0 & 0 \\ 0 & -\cos \left(\frac{\pi}{q}\right) & 1 & -\cos \left(\frac{\pi}{r}\right) & 0 \\ 0 & 0 & -\cos \left(\frac{\pi}{r}\right) & 1 & -1 \\ 0 & 0 & 0 & -1 & 1\end{array}\right]$

As a consequence, plane $b^{3}$ and the polar plane $a_{3}$ of vertex $A_{3}$ will also be parallel, as the second graph in Fig. 1 shows.

The computer visualization of the truncated orthoschemes are given in Fig. 4.

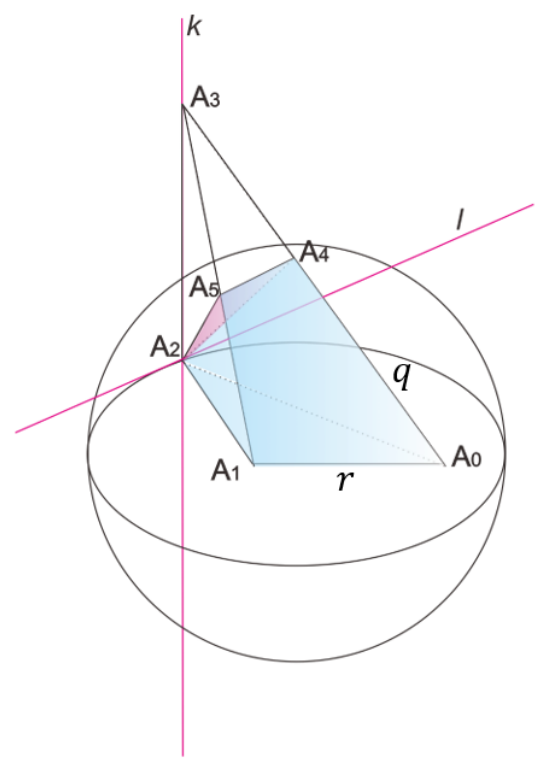

Figure 3: Truncated orthoscheme with the two intersection pairs of its parallel faces. $b^{0}$ and $b^{1}$ intersect in ideal line $k, b^{3}$ and $a_{3}=A_{2} A_{4} A_{5}$ do that in $l$. The ideal vertex $A_{2} \in k, l$. 

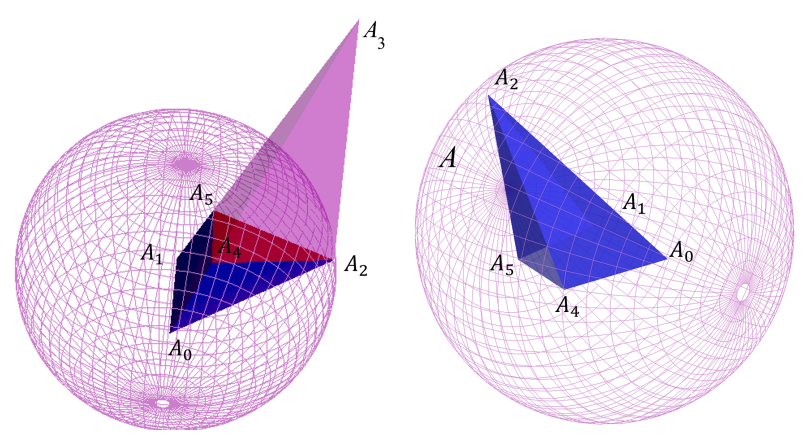

Figure 4: Truncated orthoscheme, where the truncating face is $A_{2} A_{4} A_{5}$

\section{On sphere packings}

In constructing the insphere, the largest inscribed classical sphere, in the truncated orthoscheme, we followed in [24] the procedure of [8] by bisector hyperplane.

The visualization of the optimum insphere in truncated orthoscheme $(\infty, 3,3, \infty)$ is given in Fig. 5 . The problem may occur if the insphere intersect the truncating hyperplanes $a_{3}$ (see Fig 6).

The complete packings densities of insphere packings (and their optimum density) can be found in [24], that gave the optimum packing density $\approx 0.2623649$, attained by sphere packing in $(\infty, 3,3, \infty)$, small enough, not relevant, related to [17].

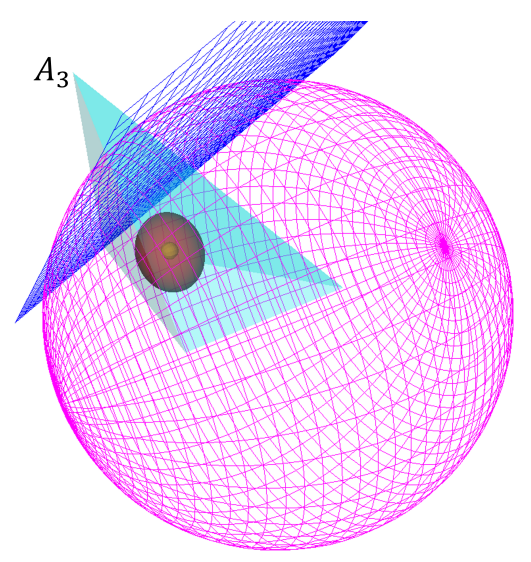

Figure 5: Optimum insphere in the truncated orthoscheme $(\infty, 3,3, \infty)$

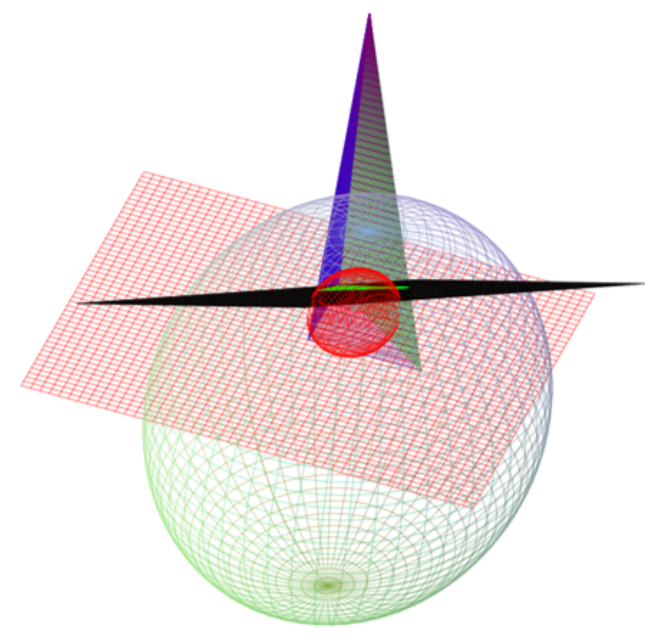

Figure 6: The insphere intersects the truncating polar plane $a_{3}$ of vertex $A_{3}$

\section{On horosphere packings}

A horosphere in hyperbolic geometry is the surface orthogonal to the set of parallel lines, passing through the same ideal point on the absolute quadratic surface (simply absolute) $\partial \mathbb{H}^{n}$ (at present $n=3$ ).

We introduce Cartesian homogeneous projective coordinate system using vector basis $\mathbf{e}_{i}(i=0,1,2,3)$ for $\mathcal{P}^{3}$ where the coordinate centre of the model is $O=(1,0,0,0)=\mathbf{e}_{0}$. We pick an arbitrary point at infinity $A_{2}=(1,0,0,1)$.

As it is known, the equation of a horosphere with centre $A_{2}=(1,0,0,1)$ through point $S=(1,0,0, s)(s \in(-1,1))$ is

$$
\frac{(s-1)^{2}}{1-s^{2}}\left(-x^{0} x^{0}+x^{1} x^{1}+x^{2} x^{2}+x^{3} x^{3}\right)+\left(x^{0}-x^{3}\right)^{2}=0
$$

This surface can be described in the usual Cartesian coordinate system by the formula

$\frac{2\left(x^{2}+y^{2}\right)}{1-s}+\frac{4\left(z-\left(\frac{s+1}{2}\right)\right)^{2}}{(1-s)^{2}}=1$,

where $x=\frac{x^{1}}{x^{0}}, y=\frac{x^{2}}{x^{0}}, z=\frac{x^{3}}{x^{0}}$.

In computer visualization, it is very powerful to convert the horosphere equation into a polar coordinate system. We use the following conversion

$x=\sqrt{\frac{1-s}{2}} \cos \theta \sin \phi, \quad y=\sqrt{\frac{1-s}{2}} \sin \theta \sin \phi$,

$z=\frac{1+s}{2}+\frac{1-s}{2} \cos \phi$

where parameters $\theta \in[0,2 \pi), \phi \in[0, \pi]$. 
We will apply the previous truncated orthoscheme (based on the set of unit normal Napier cycles) in [7, 24] as before.

We separate our discussion into two cases depending on the number of vertices lying at infinity $A_{0}, A_{2}$ or both. We can also attach two horospheres altogether, where they are touching each other on edge $A_{0} A_{2}$.

\subsection{Packings with one horosphere}

We have some truncated orthoschemes given with Schläfli symbols such that it has only one point at the infinity: $(\infty, 3,3, \infty),(\infty, 3,4, \infty),(\infty, 3,5, \infty),(\infty, 4,3, \infty)$, $(\infty, 5,3, \infty)$. However, if the truncated orthoscheme has two ideal vertices of truncated orthoscheme we can also study the corresponding horosphere packing centred at one either of these vertices.

It is clear that the densest horoshpere packing configuration would be reached whenever this horosphere (horoball) with centre $A_{2}$ touch the opposite face (represented by hyperplane $b^{2}$ ). One could simply take the projection of $A_{2}$ into $b^{2}$ by the projection formula $\mathbf{a}_{2} \longmapsto a_{2}^{p}=\mathbf{a}_{2}-\left\langle\mathbf{a}_{2}, \mathbf{b}^{2}\right\rangle \mathbf{b}^{2}$. The optimal horosphere should contain the point $A_{2}^{p}$ therefore we can determine the parameter $s$ and so the actual equation (4) of the horosphere.

We provide the computer visualization of optimum horospheres packing, attained by truncated orthoscheme tilings with Schläfli symbols $(\infty, 3,3, \infty)$, in Fig. 7-9. The optimum packing density is $\approx 0.8188080$, see [24].

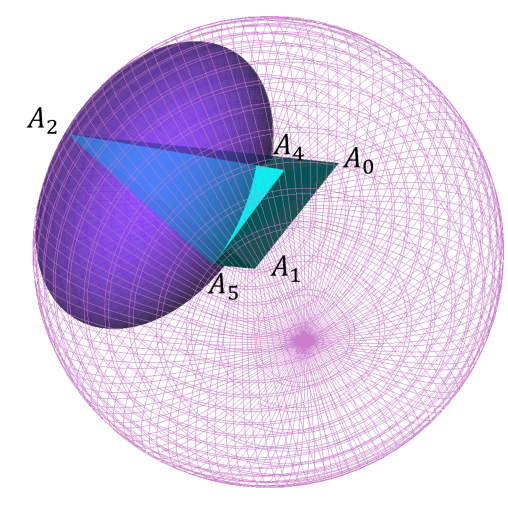

Figure 7: The largest horoball related to truncated orthoscheme of tiling $(\infty, 3,3, \infty)$

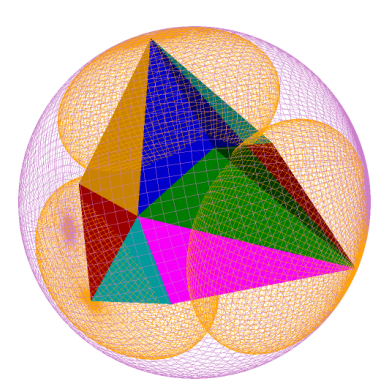

Figure 8: The neighbouring turncated orthoschemes to horosphere configuration (first crown) to tiling $(\infty, 3,3, \infty)$

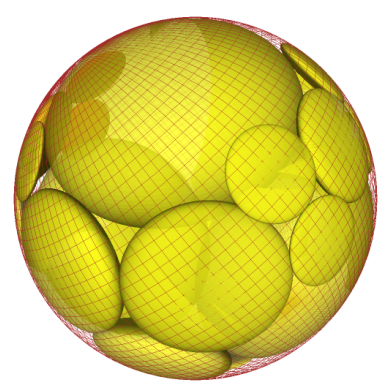

Figure 9: The first to third crowns of neighbouring horosphere configurations to tiling $(\infty, 3,3, \infty)$

\subsection{Packing with two horospheres}

Now, we focus on the orthoscheme tiling with the Schläfli symbols $(\infty, 3,6, \infty),(\infty, 4,4, \infty)$, and $(\infty, 6,3, \infty)$.

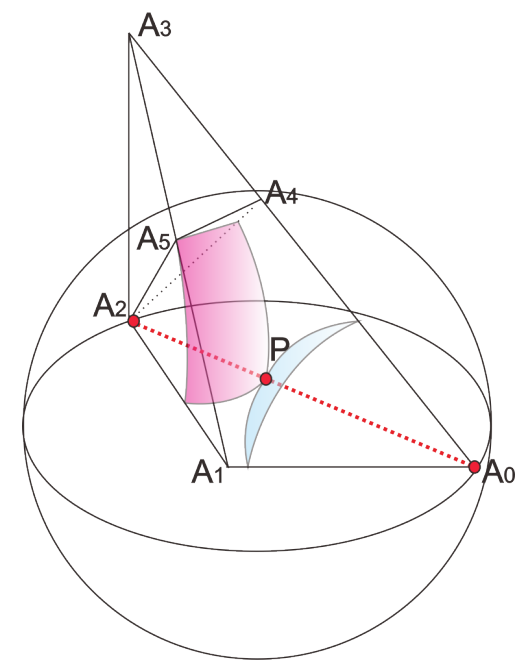

Figure 10: Two horospheres, $\mathcal{B}_{0}$ and $\mathcal{B}_{2}$, that touch each other at a point lying on edge $A_{0} A_{2}$ 
Remark 1 : These two horospheres could not intersect the opposite faces $b^{0}$ and $b^{2}$, therefore there will be a restriction for the movement of the point of tangency along edge $A_{2} A_{0}$.

We can parameterize the possible movement of the point of tangency $P$ along edge $A_{2} A_{0}$, see Fig. 10, e.g. $P(\mathbf{r}(t))=(1-t) \mathbf{a}_{2}+t \cdot \mathbf{a}_{0}$. Then, for every possible $t$, we have parameters $s_{i}(i=1,2)$ related to both horospheres.

\section{Optimal horoball packing of tiling $(\infty, 4,4, \infty)$}

In this situation, we have quite interesting structure, we obtain that the possible parameter of $t$ lies in $[\approx 0.2150<$ $t<\approx 0.3497]$. We can compute the volumes of horoball sectors as the functions of $t$. It is analogous to the previous case, the volume function of horoball sectors centred at $A_{2}$ is a monotonic increasing function of $t$ if the point of tangency moving with direction to $A_{0}$ while the volume function of horoball sectors centred at $A_{0}$ is decreasing in this situation.

In this case, we proved (in [24]) that the density was increasing as a function of $t$, see Fig. 11. Furthermore, the maximum density $\delta_{\text {opt }} \approx 0.8188081$ is attained when $t$ is largest, i.e when the horosphere centred at $A_{2}$ touches the opposite face $b^{2}$.

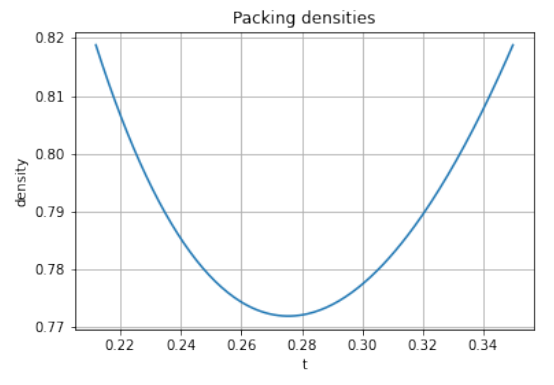

Figure 11: The plot of density function for all possible $t$ in case $(\infty, 4,4, \infty)$

Optimal horoball packing of tilings $(\infty, 3,6, \infty)$ and $(\infty, 6,3, \infty)$

We similarly visualize the densest horosphere (horoball) packings to the truncated orthoscheme tilings with Schläfli symbol $(\infty, 3,6, \infty)$ and $(\infty, 6,3, \infty)$.

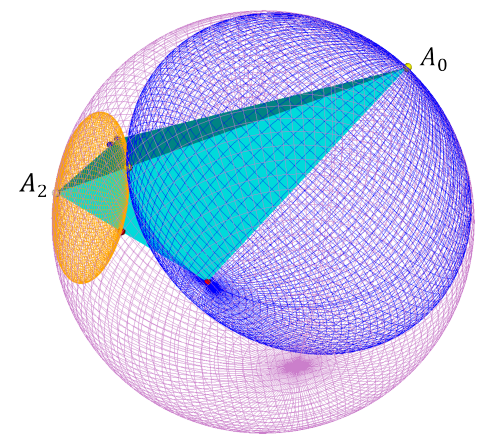

Figure 12: Two horospheres, $\mathcal{B}_{0}$ and $\mathcal{B}_{2}$, that touch each other at the point lying on $A_{0} A_{2}$ related to tiling $(\infty, 3,6, \infty)$.

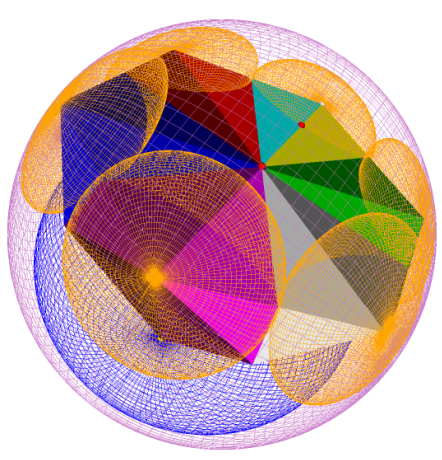

Figure 13: Adjacent orthoschemes and the corresponding horosphere configuration (first crown) to truncated orthoscheme tiling $(\infty, 3,6, \infty)$

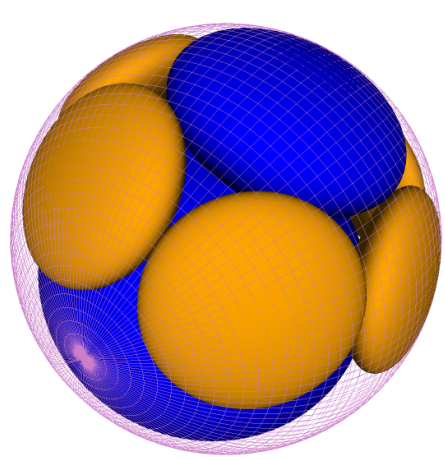

Figure 14: The horosphere configuration (first crown) related to tiling $(\infty, 3,6, \infty)$ 


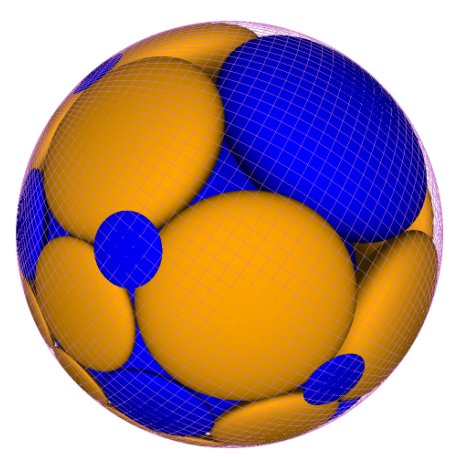

Figure 15: The optimum packing density horospheres configuration (first-third crown) to the orthoscheme tiling $(\infty, 3,6, \infty)$ with density 0.8413392 .

There are some basic facts for these (dual) orthoschemes.

1. In these symmetric dual situations, there is only one possible value of parameter $t$ in each case, $t_{(3,6)} \approx$ $0.2119416, t_{(6,3)}, \approx 0.5745582$.

2. If $(q, r)=(3,6)$ then the optimal horosphere $\mathcal{B}_{2}$ touches the plane $b^{2}$ and $\mathcal{B}_{0}$ touches the face $b^{0}$ and if $(q, r)=(6,3) \mathcal{B}_{2}$ touches the plane $b^{2}$ and $\mathcal{B}_{0}$ touches the polar face $a_{3}$.

3. The packing density of these two configurations are the same, $\approx 0.8413392$, see [24].

Finally, we give the computer visualization in Fig. 12-15 related to Coxeter tiling $(\infty, 3,6, \infty)$ and in Fig. 16-19 for Coxeter tiling $(\infty, 6,3, \infty)$.

In our opinion, non-Euclidean tilings and packings and their investigations will play an important role in the research of material structure in the near future, thus visualization of them is also important to know them better.

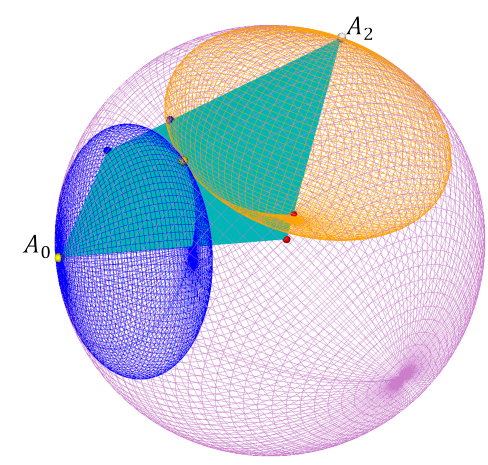

Figure 16: Two horospheres, $\mathcal{B}_{0}$ and $\mathcal{B}_{2}$, that touch each other at a point on edge $A_{0} A_{2}$ related to tiling $(\infty, 6,3, \infty)$.

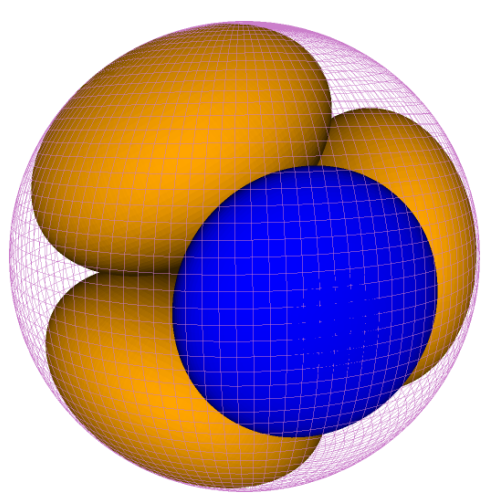

Figure 17: The horosphere configuration (first crown) to truncated orthoscheme tiling $(\infty, 6,3, \infty)$

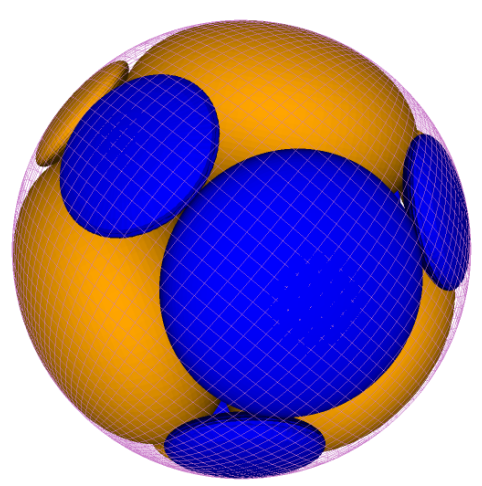

Figure 18: The optimum packing density horosphere configuration (first-second crown) related to the orthoscheme tiling $(\infty, 6,3, \infty)$.

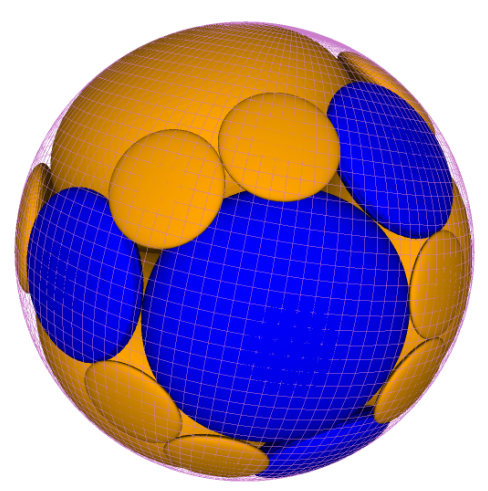

Figure 19: The optimum packing density horosphere configuration (first-third crown) to the orthoscheme tiling $(\infty, 6,3, \infty)$. 


\section{References}

[1] J. BÖHM, E. HERTEL, Polyedergeometrie in n-dimensionalen Räumen konstanter Krümmung, Birkhäuser, Basel, 1981.

[2] K. BÖRÖCZKY, Gömbelhelyezések állandó görbületü terekben I-II., Math. Lapok, 25 26, (1974, 1975).

[3] K. BÖRÖCZKY, Packings of spheres in spaces of constant curvature, Acta Math. Acad. Sci. Hungar. 32 (1978), 243-261.

[4] K. BÖRÖCZKY, A. Florian, Über die dichteste Kugelpackung im hyperbolischen Raum, Acta Math. Acad. Sci. Hungar. 15 (1964), 237-245.

[5] H. S. M. Coxeter, Regular Polytopes, Courier Corporation, 1973.

[6] L. Fejes Tóth, Regular Figures, Pergamon Press, 1964.

[7] H.-C. IM HoF, Napier cycles and hyperbolic Coxeter groups, Bull. Soc. Math. Belgique 42 (1990), 523-545.

[8] M. JACQUEMET: The inradius of a hyperbolic truncated n-simplex, Discrete Comput. Geom. 51 (2014), 997-1016.

[9] N. W. Johnson, R. Kellerhals, J. G. RatCLIFFE, S. T. TsCHANTS, The Size of a Hyperbolic Coxeter Simplex, Transformation Groups 4(4) (1999), 329-353.

[10] R. KELLERHALS, On the volume of hyperbolic polyhedra, Math. Ann. 245 (1989), 541-569.

[11] R. T. KozMA, J. SzIRMAI, Optimally dense packings for fully assymptotic Coxeter tilings by horoballs of different types, Monatsh. Math. 168(1) (2012), 27-47.

[12] R. T. Kozma, J. Szirmai, New lower bound for the optimal ball packing density of hyperbolic 4-space, Discrete Comput. Geom. (2014), doi: 10.1007/s00454014-9634-1

[13] R. T. Kozma, J. SzIRmaI, New horoball packing density lower bound in hyperbolic 5-space, Geom. Dedicata 206 (2020), 1-25.

[14] R. T. Kozma, J. SzIRmaI, Horoball packing density lower bounds in higher dimensional hyperbolic $n$-space for $6 \leq n \leq 9$, Submitted Manuscript, (2019), arXiv:1907.00595

[15] R. T. Kozma, J. SzIRmai, The structure and visualization of optimal horoball packings in 3-dimensional hyperbolic space, Submitted Manuscript, (2016), arXiv:1601.03620, (Appendix: http://homepages math.uic.edu/rkozma/SVOHP.html))
[16] E. MOLnÁR. The projective interpretation of the eight 3-dimensional homogeneous geometries, Beitr. Algebra Geom. 38 (1997), 261-288.

[17] E. Molnár, J. Szirmai, Top dense hyperbolic ball packings and coverings for complete Coxeter orthoscheme groups, Publications de L'institut Mathématique 103(117) (2018), 129-146, doi: 10.2298/PIM1817129M

[18] C.A. Rogers, Packing and Covering, Cambridge Tracts in Mathematics and Mathematical Physics 54, Cambridge University Press, 1964.

[19] J. SzIRMAI, Horoball packings to the totally assymptotic regular simplex in the hyperbolic $n$ space, Aequat. Math. 85 (2013), 471-482, doi: 10.1007/s00010.012-0158-6

[20] J. SZIRMAI, Packings with horo- and hyperballs generated by simple frustum orthoschemes, Acta Math. Hungar. 152(2) (2017), 365-382, doi: 10.1007/s10474017-0728-0

[21] J. SzIRMAI, Decomposition method related to saturated hyperball packings, Ars Math. Contemp. 16 (2019), 349-358, doi: 10.26493/1855-3974.14850b1

[22] J. SZIRMAI, The least dense hyperball covering to the regular prism tilings in the hyperbolic $n$-space, Ann. Mat. Pur. Appl. 195(1) (2016), 235-248, doi: 10.1007/s10231-014-0460-0

[23] E. B. VinberG, Geometry II: Spaces of constant curvature, Springer-Verlag, Berlin, 1993.

[24] A. YAhYA, J. SZIRMAI: Optimal ball and horoball packings generated by 3-dimensional simply truncated Coxeter orthoschemes with parallel faces, Submitted manuscript, (2021), arXiv:2107.08416.

\section{Arnasli Yahya \\ orcid.org/0000-0001-9149-7144 \\ e-mail: arnasli@math.bme.hu}

\section{Jenö Szirmai}

orcid.org/0000-0001-9610-7993

e-mail: szirmai@math.bme.hu

Budapest University of Technology and Economics Institute of Mathematics, Department of Geometry Budapest, P. O. Box: 91, H-1521 